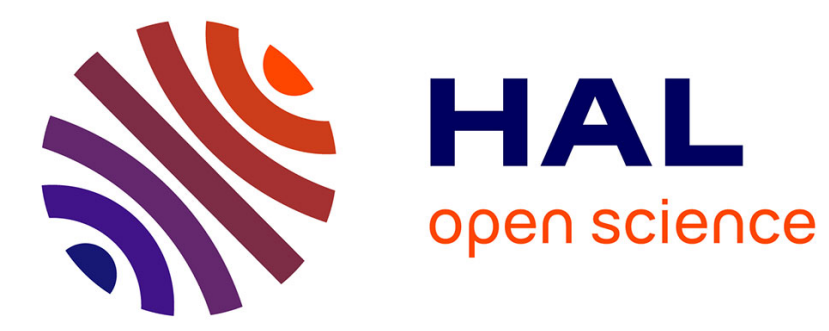

\title{
La critique du modèle perceptif de nos opérations mentales chez Thomas Reid \\ Claire Etchegaray
}

\section{To cite this version:}

Claire Etchegaray. La critique du modèle perceptif de nos opérations mentales chez Thomas Reid. Les études philosophiques, 2017, 173 (3), pp.437. 10.3917/leph.173.0437 . hal-01962650

\section{HAL Id: hal-01962650 \\ https://hal.parisnanterre.fr/hal-01962650}

Submitted on 20 Dec 2018

HAL is a multi-disciplinary open access archive for the deposit and dissemination of scientific research documents, whether they are published or not. The documents may come from teaching and research institutions in France or abroad, or from public or private research centers.
L'archive ouverte pluridisciplinaire HAL, est destinée au dépôt et à la diffusion de documents scientifiques de niveau recherche, publiés ou non, émanant des établissements d'enseignement et de recherche français ou étrangers, des laboratoires publics ou privés. 


\section{LA CRITIQUE DU MODÈLE PERCEPTIF DE NOS OPÉRATIONS MENTALES CHEZ THOMAS REID}

Au début des Recherches sur l'entendement humain d'après les principes du sens commun, parues en 1764, Thomas Reid donne une définition apparemment consensuelle de l'anatomie de l'esprit. Il lui assigne pour tâche de " considérer avec exactitude les opérations de son propre esprit et [de] les prendre pour objets de la pensée ${ }^{1} »$. Mais ce projet se heurte à plusieurs obstacles, indiqués dès les premières pages des Recherches comme plus tard, au début des Essais sur les pouvoirs de l'esprit humain publiés en $1785^{2}$. Les contourner obligera l'anatomie reidienne à repenser la notion d'opération mentale. Le premier obstacle, circonstanciel, réside dans les abus de langage et en particulier dans l'usage, par les philosophes modernes, des mots idées ou perception. Un second obstacle, plus constitutif, est lié à la complexité dans laquelle ces opérations se présentent (notamment sous l'effet de l'éducation et de la culture), ce qui rend impossible selon lui l'application de la méthode « historique " que Locke voulait suivre dans l'Essai ${ }^{3}$. D’autres difficultés, exposées au début des Essais, sont phénoménologiques. Ainsi, bien que nous ayons conscience de nos opérations, notre attention se porte habituellement à leurs objets, non à elles-mêmes ${ }^{4}$. Sur ce point, Reid rejoint Locke qui avait déclaré au tout début de l'Essai :

Comme l'œil, l'entendement nous fait voir et percevoir toutes les autres choses, mais lui-même il ne s'aperçoit pas ; aussi faut-il faire preuve d'art et d'application pour le mettre à distance et en faire pour lui-même un objet 5 .

1. Recherches sur l'entendement humain d'après les principes du sens commun (ci-après REH), I.2, trad. fr. par M. Malherbe, Paris, Vrin, 2012, p. 29.

2. Essays on the Intellectual Powers of Man (ci-après cités EIP), Édimbourg, Edinburgh University Press, 2002. Les citations seront traduites en français par nos soins.

3. Ce dernier point rend impossible l'application lockienne d'une " histoire " de l'esprit par où l'on découvrirait comment "ses facultés d'enfant commencèrent à opérer, comment elles forgèrent puis mûrirent toutes les différentes notions, opinions, ou sentiments, que nous trouvons en nous-mêmes quand nous sommes enfin capables de réflexion » (ibid., p. 31).

4. EIP, I.6, p. 60.

5. John Locke, Essai sur l'entendement humain. Livres I et II, I.i.1, tr. fr. par J-M Vienne, Paris, Vrin, 2001, p. 57. 
Mais la comparaison avec l'œil ne doit toutefois pas nous égarer. En cherchant à distinguer les opérations mentales, Reid a le souci de ne pas confondre les différentes espèces d'evidence - entendons par là les "bonnes raisons " que nous donnons naturellement de nos croyances et que les traductions classiques rendent par le terme français, dénombrable, évidences ${ }^{6}$. L'évidence perceptive doit ainsi être distinguée de l'évidence de la conscience, de l'évidence de l'induction ou encore de l'évidence démonstrative (EIP, II.20). Et à cette fin, il faut se méfier de la comparaison de toute opération mentale à une perception visuelle, comme de tous les termes philosophiques induisant un modèle perceptif de nos opérations mentales.

Par "modèle perceptif de nos opérations mentales", nous entendrons, dans ce qui suit, la réduction de toute opération mentale, qu'il s'agisse de la mémoire, de l'imagination, de la conscience ou de la perception elle-même, à la perception de quelque chose de mental (une idée ou une image par exemple). Ce modèle perceptif induit un faux problème, celui de la justification à apporter à des croyances naturelles qui n'en manquent pourtant pas car, selon Reid, elles ne requièrent d'autre justification que l'évidence (evidence) naturellement admise par les hommes. Admettre que pour se souvenir d'une chose non mentale, il faut percevoir une image d'elle ou une simple idée d'elle, n'est-ce pas laisser soupçonner que notre croyance mémorielle en une existence non-mentale passée est indue ? Et si les objets premiers de la perception sont des images ou de simples idées, comment et de quel droit la prétention à percevoir une chose non mentale serait-elle justifiée ?

Ces rappels étant faits, nous serions tentés de penser que la critique du modèle perceptif suppose de lui substituer un autre modèle. La notion d'opération pourrait suggérer de nous en tenir à un modèle pratique. Mais nous tomberions alors dans d'autres difficultés : car si se souvenir, imaginer, percevoir (etc.) c'est faire, devons-nous dire que ces opérations produisent leurs objets, soit qu'elles les fabriquent (modèle poïétique) soit qu'elles n'en aient pas en dehors d'elles-mêmes (modèle praxique) ? Un tel modèle devrait affronter le problème suivant. En identifiant l'objet de l'opération mentale à un contenu conceptuel plutôt qu'à une chose extérieure, on comprend qu'il puisse être produit par cette opération mais on ouvre la porte à une suspicion sceptique. Et la question se renverse dans le cas de l'opération spécifique qu'est l'imagination : si l'objet de nos opérations mentales n'est pas produit par elles, comment rendre compte de la conception imaginaire ? Or, ce problème, qui dépend de la façon de comprendre l'intentionnalité, est également lié à une question ontologique. On se souvient peut-être que pour rectifier l'abus de langage que les philosophes ont fait subir au terme d'idée, Reid réfère sa signification à un acte de l'esprit plutôt qu'à un objet mental. Dans le langage commun, déclare-t-il, " la pensée ne signifie rien d'autre que l'acte de penser

6. Théodore Jouffroy dans la traduction de référence comme Michel Malherbe plus récemment traduisent evidence par évidence. Cf. CEuvres complètes de Thomas Reid chef de l'École écossaise. Tome III, Paris, Sautelet et C ${ }^{\text {ie }}$, tr. fr. par Th. Jouffroy, 1828. 
(the act of thinking) et la conception, notion ou idée l'acte de concevoir (the act of conceiving $)^{7}$ ». Au XIX ${ }^{\mathrm{e}}$ siècle, Théodore Jouffroy, traduisait ici act par le français action, indiquant par là que l'opération mentale est l'exercice d'un pouvoir actif et suppose une philosophie de l'esprit qui dépasse le cadre d'une logique des actes de l'entendement. Alain De Libera, sensible à la place de Reid dans l'histoire de l'invention du sujet moderne, c'est-à-dire du sujet tenu pour agent de ses pensées, pointe l'importance de ce passage, qu'il cite dans la traduction de Jouffroy ${ }^{8}$. La question ontologique est ainsi la suivante : si une opération n'est pas une perception, est-ce à dire qu'elle est une action, c'est-à-dire l'exercice d'un pouvoir actif ? Et dans ce cas n'est-ce pas dire qu'elle est susceptible de créer son objet et peut-être même l'évidence (evidence) que nous tenons pour admise?

\section{Les opérations mentales, des actions de l'esprit?}

Si toute opération est l'action d'un agent et si c'est chez Reid que l'on voit réapparaître, comme le souligne Alain De Libera, la thèse d'un esprit agent de la pensée, ne faut-il pas admettre que cet esprit peut alors créer l'évidence, créer ses propres objets de perception et de souvenir, voire constituer ses objets? Cela semble pourtant incompatible avec la résistance de Reid au subjectivisme. Dans un manuscrit connu quoique non publié, intitulé Of Constitution, Reid défend vigoureusement que la vérité dépend non de la constitution de l'esprit connaissant mais seulement de la constitution (c'est-à-dire de la nature) des choses connues. Certes, les vérités morales tout au moins prennent l'esprit pour objet. En ce sens, elles dépendent de sa constitution. Mais pas plus que d'autres vérités elles ne dépendent de l'esprit en tant qu'agent de connaissance?

La nécessité d'affronter le problème ontologique est plus impérieuse encore si l'on souscrit à une interprétation "pragmatiste » du sens commun de Reid ou si l'on éclaire sa position en le rapprochant des auteurs tels Wittgenstein ou Searle. La littérature secondaire a montré l'intérêt de ce parallèle depuis quelques années. Patrick Rysiew, par exemple, dans un article séminal pour les études reidiennes, en vient à suggérer un rapprochement entre Reid et Kant parce que le sens commun chez Reid est, d'après lui, un ensemble de premiers principes qui sont des règles de notre vie cognitive, lesquelles sont "constitutives " de notre activité cognitive au sens que Searle a pu indiquer. Ce dernier distingue en effet les règles "régulatives ", qui viennent seulement réguler un comportement pré-existant, des règles " constitutives » (comme celles du jeu d'échec) qui créent la possibilité même

7. EIP, II.9, p. 132 - cf. tr. fr. par Th. Jouffroy, in Euvres complètes, op. cit., p. 177. Reid dit la même chose de la conception en EIP, II.14, p. 184.

8. A. De Libera, Archéologie du sujet. La double révolution, Paris, Vrin, 2014, p. 604.

9. Th. Reid, "Of constitution ». Thomas Reid Papers, MS 3061/8. Aberdeen, University's Digital Repository. 
d'une activité. Rysiew souligne que chez Reid, les premiers principes créent de la même façon la possibilité d'une activité mentale ayant une certaine valeur : la connaissance ${ }^{10}$. En outre ces règles ne sont pas de simples régularités. Elles ont, comme celles que Searle analysait, un caractère normatif. Par exemple, à propos de la perception, une simple régularité serait susceptible d'être énoncée dans une phrase telle qu'il se trouve quion croit toujours quily a un objet hors de notre esprit lorsqu'on perçoit, alors que le premier principe de Reid concernant la perception est que les choses que nous percevons distinctement par nos sens existent réellement et sont telles que nous les percevons. Ces règles sont donc indissociablement normatives et descriptives. Rysiew y voit là une raison de rapprocher Reid et Kant ${ }^{11}$. Mais un tel parallèle interroge : l'objet des opérations mentales ou la garantie de nos croyances qu'est l'evidence sont-ils chez Reid "constitués " par l'action du sujet pensant ${ }^{12}$ ?

Notons d'abord que Reid fait deux emprunts notables à la philosophie médiévale (tous deux soulignés par Alain De Libera) pour conceptualiser l'action de penser, emprunts qui permettent également de nuancer l'attribution à Reid d'un modèle poḯtique ou praxique : la "dénomination extrinsèque " et la distinction entre "acte immanent et acte transitif " ${ }^{13}$. Ainsi, être perçu est une dénomination extrinsèque, c'est-à-dire qu'elle n'affecte aucunement la chose perçue ${ }^{14}$. En outre, il faut distinguer les opérations intellectuelles, immanentes, des opérations pratiques et volontaires, transitives. Reid s'oppose ici en particulier à la théorie de la perception proposée par Newton et Clarke qui fait l'hypothèse d'un sensorium où l'âme est présente aux images et les images à l'âme. Clarke justifie cette hypothèse dans sa correspondance avec Leibniz en disant que l'âme ne peut ni agir ni être agie où elle n'est pas ${ }^{15}$. La perception, dans ce cadre, est soit l'action des choses sur

10. J. R. Searle, Speech Acts. An Essay on the Philosophy of Language, Cambridge University Press, 1969, p. 34-35.

11. P. Rysiew, "Reid and epistemic naturalism ", Philosophical Quarterly 52 (209), 2002, p. 437-456 et "Pragmatism and Reid's "Third Way" ", Thomas Reid on Mind, Knowledge and Value, Oxford University Press, 2015, p. 178-192. Cf. également Ameriks K., Kant and the Historical Turn, Oxford New York, Oxford University Press, 2006.

12. Angélique Thébert fait un rapprochement avec Wittgenstein lorsqu'elle défend que le " track-record argument " de Reid vise moins à donner une justification à des croyances qui n'en auraient pas qu'à attester de la maîtrise d'un savoir-faire en quoi consiste la connaissance humaine. Elle cite notamment ce texte : "Giving grounds, however, justifying the evidence, comes to an end ; - but the end is not certain proposition's striking us immediately as true, i.e. it is not a kind of seeing on ou part ; it is our acting, which lies at the bottom of the languagegame " (De la certitude, $\$ 204$, cité par A. Thébert dans "The defence of the first principles of common sense in Reid's epistemology ", in Thomas Reid on Mind, Knowledge \& Value, éd. R. Copenhaver et T. Buras, Oxford, Oxford University Press, 2015, p. 200).

13. EIP, II.14, p. 177 (cf. EIP, II.4, p. 89).

14. On se souvient que Descartes avait déjà utilisé cette notion médiévale dans sa réponse aux objections de Caterus. Descartes, Réponses aux premières objections in Euvres philosophiques. 1638-1642, éd. F. Alquié, Paris, Classiques Garnier, 1992, volume II, p. 129.

15. Clarke déclare dans la seconde réponse à Leibniz : "Il n'est pas possible que l'âme, si elle n'est présente aux images des choses perçues, puisse les percevoir. Une substance vivante peut seulement percevoir au lieu où elle est présente, soit dans les choses elles-mêmes (comme Dieu omniprésent l'est à tout l'univers), ou dans les images des choses, comme l'âme l'est dans son propre sensorium." (Seconde réponse de Clarke, décembre 1715, section IV, in 
nous, soit l'action de l'âme sur les choses. Or le fait d'être perçu pour la chose ne suppose aucune action en elle; et le fait de la percevoir ne lui fait rien non plus. En outre toutes nos opérations intellectuelles sont des actes immanents, qui "ne produisent aucun effet hors de l'esprit». Ainsi, percevoir, se souvenir, prendre conscience ne sont pas des actions impliquant un rapport entre l'esprit et l'objet qui soit l'activité d'un agent sur un patient (que ce soit de l'esprit sur l'objet ou de l'objet sur l'esprit). Mais alors quel sens donner à cette notion d'opération de l'entendement?

On a dit plus haut que Jouffroy avait des raisons de traduire, à l'occasion, act of mind par " action de l'esprit ». C'est que tout pouvoir est au sens strict un pouvoir actif d'après Reid et par conséquent, toute opération, dans la mesure où elle est l'exercice d'un pouvoir actif est une action. Cette dernière thèse semble parfaitement consonante avec la thèse, exprimée dans les Essais, selon laquelle l'esprit, qui est le "sujet " des opérations, " de par sa nature même » est " un être actif» (p. 20-21). Toutefois, ainsi que Laurent Jaffro l'a souligné, dans les opérations intellectuelles l'esprit est seulement dit « exercer quelque degré d'activité » (EIP p. 65). Elles ne sont volontaires que dans la mesure où l'esprit peut exercer sa perception, sa mémoire, sa conscience avec plus ou moins d'attention. Mais dans la mesure où ce sont des faits de nature, des parts of our constitution, elles sont instinctives et non volontaires. Ainsi, au sens strict, ce ne sont pas des actions de l'esprit, ce sont des actions de Dieu en l'esprit ${ }^{16}$. Ce point semble nuancer la lecture faite par Alain De Libera et la traduction de Jouffroy. Mais c'est au sein du texte reidien que le problème se pose et doit être résolu. De quel droit dire que "je réalise " les opérations mentales (I really perform all those operations of which I am conscious) comme Reid le fait dès le premier des principes tenus pour admis qu'il présente en préliminaire de la lecture des $E_{s s a i s^{17}}$ ? De quel droit dire que c'est l'esprit qui est le «sujet » (subject) et l'" agent » (agent) de " toutes » les opérations?

Les pouvoirs, facultés et opérations de l'esprit sont des choses en l'esprit. Toute chose est dite être dans l'esprit, dont l'esprit est le sujet (EIP, p. 21-22).

Nous croyons, par conséquent, à titre de premier principe [...] que toute opération de l'esprit dont nous avons conscience suppose un agent de ces opérations, que nous appelons esprit (EIP, p. 44).

Correspondance Leibniz-Clarke, présentée d'après les manuscrits originaux des bibliothèques de Hanovre et de Londres, par André Robinet, Paris, Puf, 1957, p. 48); puis dans la troisième réponse à Leibniz: "Nous sommes certains que l'âme ne peut pas percevoir ce qui ne lui est pas présent, parce que rien ne peut agir ou être agi où il n'est pas " (Troisième réponse de Clarke, mi-avril 1716, section XI, in Correspondance Leibniz-Clarke, op. cit., p. 71). Newton et Clarke excluent que la perception puisse être une forme d'action à distance.

16. L. Jaffro, "Reid on powers of the mind and the person behind the curtain", in Canadian Journal of Metaphysics, vol. 41, $\mathrm{n}^{\circ} \mathrm{S1}, \mathrm{p} .197-213$.

17. EIP, p. 41 : «I really perform all those operations of mind of which I am conscious ». Notons toutefois que dans ce passage Reid est occupé à affirmer non l'existence d'un moi, mais celle des opérations mentales. Ici, really indique la réalité des opérations dont on a conscience et connote la fiabilité de la conscience. 
Pour répondre à ces questions, il faut se souvenir que Reid n'emploie pas la notion de sujet au sens de l'agentivité, ni même au sens moderne de la subjectivité, mais seulement au sens d'un sujet d'inhérence et d'attribution, tel qu'on le trouve au Moyen-Âge. Le corps est ainsi le sujet des propriétés que nous percevons et l'esprit est celui des opérations dont nous avons conscience - c'est-à-dire ce à quoi nous attribuons les propriétés ou les opérations ${ }^{18}$. C'est à l'aune de ce seul sens, également, qu'il faut comprendre selon Reid la distinction entre choses internes et choses externes : il y a des choses dont l'esprit est le sujet d'attribution (et elles sont dites "internes") et il y a des choses dont il ne peut être le sujet d'attribution (elles sont alors dites « externes », ce sont les corps.). Il est vrai que Reid prête en outre attention à la croyance naturelle d'après laquelle l'esprit est un self, et il l'admet comme un présupposé incontournable également en philosophie ${ }^{19}$. La conscience de nos opérations mentales s'accompagne de la conviction que c'est l'esprit, en première personne, qui a le pouvoir de les exercer. Mais l'anatomiste de l'esprit n'étudie pas l'esprit en tant que self. Il l'étudie en tant que sujet, de même que, pour le philosophe de la nature, les corps sont des sujets de propriétés. Et le logicien, dont la tâche, est d'après Reid de " présenter la nature de l'évidence (evidence) et d'en distinguer les divers degrés et espèces ", ne s'en acquitte pas en démontrant que parce que l'esprit est un self ayant une certitude de soi il peut ensuite en venir, comme l'espérait Descartes, à acquérir une certitude sur les autres choses; il doit plutôt considérer chaque cas d'evidence comme un fait de nature (c'est-à-dire la valeur cognitive de la perception, de la mémoire, de la conscience, etc.).

Il y a "opération" lorsque l'effet produit (dont on a l'expérience) est l'effet d'un pouvoir détenu par l'esprit - un pouvoir qui ne peut pas être détenu par le $\operatorname{corps}^{20}$. Reid s'oppose d'ailleurs à toute théorie des opérations mentales qui donnerait un rôle explicatif à la matière ou encore à des impressions des objets externes ${ }^{21}$.

Si l'on devait dire d'un télescope si précisément construit qu'il a le pouvoir de voir, d'une galerie à écho qu'elle résonne tant et si bien qu'elle a le pouvoir d'entendre,

18. Sans avoir pour objectif d'examiner la position ontologique de Reid comme telle, Alain De Libera rappelle souvent que les deux sens de la notion de sujet (subjectität et subjectivität) sont bien distincts, même s'ils ont pu se rejoindre. L'ensemble de ses travaux de l'Archéologie du sujet cherche à retracer comment cette rencontre a pu être possible à la période moderne. Il retrace en outre les origines médiévales de la notion reidienne de dénomination extrinsèque.

19. "Lorsque je dis : je vois, j'entends, je sens, je me souviens, cela implique qu'il y a un seul et même moi (self) qui opère (performs) toutes ces opérations, et comme il serait absurde de dire que ma mémoire, l'imagination d'un autre homme et la raison d'un troisième homme peuvent faire un être intelligent individuel, il serait également absurde de dire qu'un morceau de matière doué de la vision, qu'un autre doué de l'ouïe et qu'un troisième doué du toucher constituent un seul et même être percevant (percipient being)" (EIP, II.1, p. 73).

20. " $[\mathrm{L}]$ a nature du corps est telle quil ne peut changer d'état que par quelque force imprimée sur lui. Telle n'est pas la nature de l'esprit. Tout ce que nous savons de lui montre qu'il est de sa nature d'être vivant et actif, et d'avoir en sa constitution le pouvoir de perception, mais toujours dans les limites fixées par les lois de la Nature »(EIP, II.4, p. 89-90).

21. Cf. EIP, II.3. 
d'un cabinet si bien meublé qu'il a un pouvoir de mémoire ou d'une machine si sensible qu'elle a le pouvoir de sentir la douleur quand elle est touchée, ces absurdités seraient si choquantes pour le sens commun qu'elles ne rencontreraient la croyance de personne, même parmi les sauvages. Cependant il est tout aussi absurde de penser que les impressions des objets externes sur la machine de nos corps peuvent être la véritable cause efficiente de la pensée et de la perception (the real efficient cause of thought and perception) (p. 88).

Dire qu'un télescope ingénieusement arrangé voit, qu'une galerie à écho entend, qu'un cabinet de curiosité conserve en mémoire (etc.) c'est oublier le sens de l'opération*. Par définition, toute opération de l'esprit se fait en première personne. Puisque les objets de la conscience sont limités à nos opérations mentales, on peut définir l'opération mentale comme ce dont on a conscience et on peut dire que la conscience c'est l'expérience même de nos opérations mentales en première personne.

Venons-en à la notion d'agent. Elle peut désigner plusieurs choses : ce qui détient un pouvoir d'agir et peut causer un certain effet, ce qui est cause de ce pouvoir même ou bien encore la cause de son exercice. L'esprit détient assurément des pouvoirs intellectuels tels que ceux de voir, se souvenir, raisonner, etc. Comme ces pouvoirs s'exercent en lui, l'esprit est sujet (d'attribution) de ses opérations intellectuelles (EIP I.1, p. 21). Qu'il en soit l'agent est une conviction naturelle. Mais l'esprit n'est pas la cause active de l'existence et de la nature de ce pouvoir de percevoir, de se souvenir ou de raisonner. Il ne peut être nommé cause active car ce terme renvoie à l'action d'un créateur. Il n'est pas non plus agent libre d'exercer ou non cette capacité dans certaines conditions. Par exemple, telle sensation est signe de telle qualité de l'objet - ce lien de signification, l'esprit n'en décide pas : c'est un fait, et c'est cela, percevoir ${ }^{22}$. Alors, l'esprit est-il l'agent du pouvoir qu'il met en œuvre dans l'opération mentale? Rien n'est moins sûr. Reid dit certes que l'esprit est un "living and active being" (EIP, I.1 p. 21 ; p. 35). Mais qu'est-ce à dire? L'esprit est un être actif au même titre qu'il est un être vivant. De même que l'esprit n'est assurément pas le créateur de la vie, quoiqu'il en soit le sujet ou le principe dans l'être organique, de même il n'est pas à l'origine du pouvoir mental qu'il utilise mais il est le principe ou, au sens attributif, le sujet de ce qui est réalisé par là et que l'on nomme opération mentale. Un "être actif " pour Reid c'est un être qui possède un pouvoir actif et par ce pouvoir peut produire un changement en lui-même. Mais pour autant il n'est pas nécessairement la cause active de ce pouvoir. Reid d'ailleurs fait la différence entre avoir conscience de ses opérations mentales et avoir conscience de l'activité

* Travers dans lequel sont tombées les théories matérialistes de Hobbes à Hartley.

22. Par ailleurs, l'esprit a un pouvoir d'attention qui "peut être nommé volontaire " $(E A P$, II.3, p. 77). C'est par là qu'il peut acquérir un contrôle sur ses capacités naturelles. Ainsi, une forme de négligence des sensations conduit à empêcher la suggestion naturelle d'opérer; à l'inverse, c'est en prêtant attention à des sensations peu vives que la perception s'exerce plus finement. 
qui les cause : nous avons certes conscience des opérations mentales, mais pas de la cause active qui les produit ${ }^{23}$.

Ne pourrait-on dire tout au moins que l'esprit est la " cause active " d'une opération mentale ? En un sens il est bien actif puisqu'il a un pouvoir et que ce pouvoir s'exerce à la première personne, mais il n'est pas la cause efficiente de ce pouvoir (de la possibilité de l'exercer ou non dans certaines conditions données, par exemple lorsque telle sensation ou tel signe se présentent). Reid dit parfois que nous avons un pouvoir et que l'action de Dieu consiste à nous l'avoir donné et à lui assigner des limites ${ }^{24}$. Mais il répète souvent aussi que nous sommes « déterminés par notre constitution " à tenir la vérité d'un axiome ou d'un principe pour accordée. En somme l'exercice de ce pouvoir qu'est l'opération intellectuelle n'est pas libre. (Il en va autrement de l'exercice de ce que Reid nomme nos pouvoirs actifs, parmi lesquels on compte le pouvoir d'attention, ou encore les pouvoirs d'agir sur les choses, dont les opérations sont donc transitives).

Pour finir sur ce point, il est possible de distinguer l'opération mentale de la propriété corporelle. La propriété corporelle est perçue, induite par observation; l'opération mentale fait l'objet d'une expérience propre par la conscience, et on y prête attention par réflexion. Celle-ci est l'attention portée à nos opérations. C'est une intuition. La propriété appartient à une chose, qui est un corps; l'opération mentale appartient à un esprit, qui en a conscience. En outre, l'opération mentale a un objet qui en est distinct. Plus exactement, la plupart de nos opérations mentales ont des objets distincts d'elles-mêmes. La nuance "la plupart" tient au fait qu'il y a au moins une sorte d'opération mentale où l'objet n'est pas distinct de l'opération : la sensation, qui est à elle-même son propre objet. Mais alors nos questions se posent à nouveau : est-ce à dire que "la plupart» des opérations créent leurs objets ou encore qu'elles sont à elles-mêmes leurs objets, des objets mentaux donc? Si ce que nous avons dit est juste, il faut, pour répondre à la première question insister sur le fait qu'il ne s'agit pas de dire que l'esprit humain produit par lui-même son objet. C'est l'ensemble des lois de nature de notre constitution mentale, instituée par son créateur, qui fait que nos opérations ont tels ou tels objets. Pour répondre à la seconde question il va falloir examiner la notion "d'objet». Comme telle l'assertion selon laquelle «la plupart de nos opérations mentales ont un objet qui en est distinct " n'est pas encore

23. Reid d'ailleurs fait la différence entre avoir conscience de ses opérations mentales et avoir conscience de l'activité qui les cause : nous avons certes conscience des opérations mentales, mais pas de la cause active qui les produit. $C f$. les commentaires de Reid sur l'Essay on the Difference between the Relation of Motive and Action, and That of Cause and Effect, in Physics de James Gregory, in The Correspondance of Thomas Reid, Édimbourg, Edinburgh University Press, 2002, p. 252.

24. "Nous percevons, parce que Dieu nous a donnés le pouvoir de percevoir et non parce que nous avons des impressions tirées des objets. Nous ne percevons rien sans ces impressions, parce que notre Créateur a limité et circonscrit nos pouvoirs de perception par les lois de Nature qui semblent convenir à sa sagesse et qui sont adaptées à notre rang dans sa création " (p. 95). 
une thèse ontologique. Ce n'est pas une déclaration de réalisme direct. C’est simplement la thèse (encore assez vague et indéterminée, semble-t-il) que toute opération mentale est de quelque chose, qui en est distinct. Par exemple, lorsque je me souviens de quelque chose passée, mon souvenir est présent. Ce point est d'abord avancé comme un principe tenu pour admis. Mais dans l'anatomie de l'esprit qu'il propose, Reid va mettre en évidence un fait appartenant à toute opération mentale : la conception, à propos de laquelle la question ontologique croise la question de l'intentionalité.

\section{La conception. Toutes les opérations intellectuelles ont un objet.}

Le terme d'intentionalité n'est pas employé par Reid. Pour nous concentrer sur les difficultés soulevées par la pensée de Reid sans y projeter de catégorie étrangère, nous tenterons d'élucider la notion d'objet sans recourir à la catégorie, absente chez Reid, d'objet intentionne ${ }^{5}$.

Ce qui, dans l'opération mentale, consiste à penser à quelque chose et donc à penser à un objet, c'est ce que Reid nomme d'un terme qu'il reprend à la tradition mais sans souscrire aux thèses classiques qui l'accompagnaient, la conception ${ }^{26}$. Celle-ci ne préexiste pas à l'opération - elle en est l'ingrédient. Elle peut toutefois être détachée de l'opération et se trouver "nue » : elle est alors une simple apprehension ou bare conception, obtenue par abstraction ${ }^{27}$. Mais dans le cas d'une simple conception, n'y a-t-il pas un objet mental ? N'est-ce pas l'indice que dans toute conception, donc toute opération, ce qui

25. Dans « Reidian Intentionality " (Reid studies, vol. V, Autumn 2001, p. 53-64), JohnChristian Smith tente pour sa part de dégager une conception reidienne de l'intentionalité en rectifiant la caractérisation de l'intentionalité que donne Chisholm par des thèses reidiennes sur nos opérations mentales. Smith fait peu de cas de la critique reidienne de la théorie des idées, laquelle, comme on l'a vu plus haut, n'est pas sans susciter un paradoxe pour la compréhension de l'intentionalité et il répète à l'envie que l'objet intentionnel est intrinsèque ou immanent à l'opération mentale. Or, selon Reid leurs objets ne sont pas intrinsèques ou immanents à l'opération mentale, puisqu'ils sont distincts. En outre, prenant pour admis qu'il y a une intentionalité reidienne, insiste sur la distinction entre l'acte et l'objet intentionnel. Mais le véritable défi, pour le lecteur contemporain de Reid, est de comprendre comment Reid parvient à rendre compte du fait que toute opération mentale a un objet, tout en niant que l'objet de l'imagination soit une image distincte de l'acte et que l'objet de la conception abstraite soit un objet mental universel - et cela, enfin, sans convoquer la notion d'objet intentionnel. Il est vrai que Reid dit que la conception est un acte "immanent » en ce qu' elle ne produit pas un effet distinct d'elle - mais c'est l'acte qui est immanent, pas son objet, lequel, dans la perception, le témoignage, la conscience, est ce qui existe, un attribut d'une chose matérielle ou d'un esprit déjà-là.

26. EIP, V.1, p. 296.

27. Cela ne signifie pas, pour autant, que le contenu de la simple conception est le même que le contenu de la conception perceptive (comme si, par exemple, elle restait de la perception une fois qu'on en soustrait la croyance). Sur ce point, on aurait pu souhaité que Reid précise davantage la différence entre la conception imaginaire et la conception impliquée par la perception, comme le regrette Marina Folescu (" Perceptual and imaginative conception. The distinction Reid missed ", in Thomas Reid on Mind, Knowledge and Value, Oxford, Oxford University Press, 2015, p. 52-74). 
est présent à l'esprit est un objet de l'esprit ? Précisons donc ce que peut être l'objet d'une conception.

1. D'abord ce peut être l'objet de l'opération mentale où la conception est impliquée. Par exemple, l'objet de la conception impliquée dans la perception c'est la propriété ou la qualité corporelle; l'objet de la conception impliquée dans la conscience c'est l'opération mentale. Plus largement, l'objet peut désigner une chose qui existe (un esprit ou un corps, donc) et à laquelle on attribue toutes ces qualités ou propriétés, ces opérations ou pouvoirs.

Beaucoup d'auteurs ont observé que, lorsque nous concevons simplement un objet, les ingrédients de cette conception doivent être des choses que nous avons découvertes (with which we were before acquainted) par quelque autre pouvoir original de l'esprit, ou doivent être des parties ou des attributs de ces choses (EIP, IV.1, B 308).

Les objets, en ce sens, sont donc les choses matérielles dont nous percevons les propriétés et les esprits qui agissent, c'est-à-dire qui ont des opérations mentales pour attributs.

2. Mais qu'en est-il des "simples conceptions " de l'imagination ou des conceptions abstraites ? Il faut déjà souligner que l'objet de la simple conception imaginaire n'est pas une image produite par la conception, contrairement à une analogie naturelle que nous sommes tentés de faire entre la conception d'un côté et la peinture ou les pouvoirs plastiques de l'autre. Cette analogie nous fait considérer la conception comme un " pouvoir plastique, par lequel nous formons pour nous-mêmes des images des objets de la pensée ${ }^{28}$ ". Cela nous suggère que l'image serait distincte de l'acte d'imaginer. Or, l'image n'est pas un effet qui existe indépendamment de l'action d'imaginer. Pour le montrer Reid se sert une nouvelle fois de la distinction médiévale entre acte immanent et acte transitif :

Lorsqu'un homme peint, il y a quelque œuvre réalisée, qui demeure lorsqu'il enlève ses mains et qui continue d'exister, bien qu'il n'ait plus à y penser. [...] Qu'est-ce que la conception ? C'est un acte dans l'esprit de celui qui opère. On ne saurait le nier. Mais produit-il un effet quelconque en dehors de l'acte lui-même? Assurément le sens commun répond à cette question par la négative. Car chacun sait que c'est une chose de concevoir, une autre de mener quelque chose à sa réalisation effective. C'est une chose de projeter, une autre d'exécuter. Un homme peut penser pendant longtemps à ce qu'il a à faire et finalement ne rien faire. Concevoir aussi bien que projeter ou se résoudre à sont ce que les scolastiques nomment des actes immanents de l'esprit, qui ne produisent rien en dehors d'eux-mêmes. Mais peindre est un acte transitif, qui produit un effet distinct de l'opération et cet effet est l'image peinte (picture). Rappelons-nous toujours, par conséquent, que ce qui est communément nommé l'image (image) d'une chose dans l'esprit n'est rien de plus que l'acte ou l'opération de l'esprit qui la conçoit (IP, IV.1, B 300).

28. EIP, IV.1, p. 299. 
Concevoir ce n'est pas produire une chose existant indépendamment de la pensée - c'est produire un effet qui n'existe que tant qu'on pense, un effet qui n'existe pas en dehors de l'acte lui-même. Quand on parle d'image pour désigner une conception, par conséquent, ce n’est pas au sens pictural de ce qui a été produit par un acte et peut avoir une existence séparée ou indépendante de lui. Le modèle plastique, qui est un modèle poïétique pictural, est donc exclu, c'est pourquoi la conception n'a pas d'objet propre : elle a l'objet des opérations mentales où elle est impliquée. Si l'image pouvait exister indépendamment de l'acte d'imaginer, elle serait l'objet d'une conception imaginaire. Or l'image n'est pas l'objet de la conception imaginaire. De même que chacun peut faire la différence entre concevoir un cheval et concevoir l'image d'un cheval, il est possible selon Reid de distinguer la conception d'un centaure que l'on a en y pensant et la conception de l'image d'un centaure qui naît de la contemplation d'une toile classique. Si l'on veut parler d'image, à propos de l'imagination d'un centaure, on doit la considérer non comme l'objet de la représentation mais comme la représentation même. Elle "n'est pas non plus un effet produit par la conception comme par une cause ", précise Reid ; " elle est la conception même " (EIP, IV.1, p. 301).

Limagination a pour objet une chose imaginée - mais pas une image de cette chose. Comment expliquer alors que cette simple conception ait pour objet quelque chose qui n'existe pas ? L'explication que Reid donne de la simple conception imaginaire est en réalité très empiriste. Il y a un sens de l'imagination que Reid restreint à la conception de l'objet visible : songer à un objet qu'on a déjà vu, c'est l'imaginer ${ }^{29}$. Et il y a un sens plus large de l'imagination : la fantaisie ( $f a n c y)$, i.e. la conception d'un objet non-existant qu' on n'a pas vu comme tel, et qu'on recompose à partir d'attributs déjà découverts par ailleurs ${ }^{30}$. Ainsi, il est impossible qu'un homme puisse concevoir des couleurs s'il n'en a jamais vues. Ici Reid reprend l'exemple de la missing shade of blue de Hume : la nuance manquante de bleu peut être conçue parce qu'elle n'est qu'un degré des bleus déjà vus. En somme, l'imagination est bien une conception d'objet parce qu'elle est une conception combinant d'autres conceptions d'objets (impliquées au préalable dans d'autres opérations mentales). Mais il n'est pas sûr que cela résolve toutes les difficultés : puisque les conceptions sont des actes, comment la combinaison d'actions mentales pourraient-elles faire une chimère ou un château ambulant ? En éliminant l'image mentale et même tout objet mental, et en identifiant la conception à un acte, Reid court le risque de s'empêcher de penser un contenu. Pourtant il continue de parler de choses conçues, par exemple à propos des conceptions générales (EIP, V, B 375 ou $\mathrm{B}$ 386) pour désigner ce qui est conçu dans le cas d'un air de musique conçu par son compositeur, d'un plan d'architecture qu'on n'a pas exécuté, d'un programme de gouvernement tel que l'Oceana 
d'Harington. Comment envisager ces choses qui n'existent pas encore ou pas du tout - et qui pourtant ne sont pas des choses mentales ?

Il faut admettre qu'il y a là chez Reid une difficulté, qu'il ne traite pas de façon directe et systématique. La seule solution, pour lui, est de la dissoudre. D'un point de vue ontologique, il n'y a que des choses qui ont existé, existe ou existeront (des corps et des esprits qui ont eu, ont ou auront des propriétés et des opérations). Parmi les opérations, qui existent, il y a des actes qui consistent à concevoir, c'est-à-dire des actes dont on dit qu'ils sont $d e .$. (perception $d e \mathrm{x}$, souvenir de $\mathrm{x}$, conscience de $\mathrm{y}$, imagination de $\mathrm{z}$ ). Donc l'alternative est simple : soit la chose conçue existe, soit elle n'existe pas. Si elle n'existe pas, on peut tout de même en parler - et ce qui existe alors, c'est l'acte. Toutefois, il semble que Reid lui-même se heurte aux limites de cette (dis)solution quand il demande de distinguer chose conçue et acte de concevoir ${ }^{31}$. Il le fait, bien entendu, non pour dire que la " chose conçue » est un objet mental, mais pour dire que ce de quoi la conception est conception dans le cas de la composition musicale, du projet architectural ou du plan de gouvernement, appartient au domaine des choses qui sont les objets de nos opérations mentales, même si elles n'existent pas ou pas encore ${ }^{32}$. L'espace ontologique pour des choses possibles semble se refermer : soit on peut dire qu'elles existent, mais en tant qu'actes mentaux, soit il faut dire qu' elles n'existent pas.

Reste une question : pourquoi l'analogie plastique est-elle si « naturelle »? Reid dit même qu'elle est «inévitable». Qu’est-ce qui nous pousse si naturellement à entendre dans le mot "conception" une production d'image? Le seul élément de réponse que Reid propose est le suivant :

Rien ne donne plus promptement la conception d'une chose que d'en voir une image. De là, par une figure de langue commune, on appelle la conception une image de la chose conçue (EIP, IV.1, p. 301).

Lorsque nous regardons une image, par exemple une toile de peinture ou un reflet dans un miroir, il y a une opération, la perception, qui a pour objet l'image. Celle-ci étant signe de quelque chose, nous en venons à concevoir la chose dont elle est le signe. L'image, toutefois, ne nous fait pas percevoir cette chose. Nous avons donc conscience de ne pas percevoir la chose dont elle est l'image, mais seulement de la concevoir. Et inversement, nous avons conscience de percevoir l'image elle-même, une image qui n'est pas en l'esprit, mais sur la toile ou le miroir. Ce cas est d'après Reid celui où une simple conception nous vient " le plus promptement " à l'esprit - c'est donc le cas le plus facile où l'on conçoit une conception. Car habituellement, dans les autres opérations mentales où la conception est impliquée, elle s'accompagne de croyance et porte notre attention sur un objet au sens 1 . Il nous faut faire un effort de réflexion pour la prendre pour objet. Mais dans

31. EIP, p. 386.

32. EIP, II.14, p. 172. 
le cas de la contemplation d'une image, nous avons facilement conscience de concevoir un reflet ou une peinture indépendamment d'une croyance perceptive, mémorielle ou testimoniale en la chose qui y est représentée. Ainsi dans cette expérience, on a facilement conscience de concevoir une chose représentée, en même temps que l'on a conscience de la perception visuelle d'une représentation matérielle. L'analogie se produit alors naturellement : la conception est pensée sur le modèle de la vision d'une image mentale. L'expérience de la perception d'une image matérielle est donc à la source du langage analogique.

\section{Les opérations mentales ne sont pas des perceptions mentales}

Implicitement aussi, l'exemple permet de mieux comprendre la séduction opérée par le modèle optique de la perception sur les philosophes. Il est maintenant temps de considérer en détail ce modèle erroné. Nous montrerons toutefois que le modèle perceptif incriminé n'est pas seulement visuel. Certes, le modèle optique de la perception est à l'horizon des discussions de Reid. L'influence des travaux d'optique mettant en évidence les images cérébrales dans la perception sur les théories de la perception de Locke, Newton et Clarke a été par ailleurs établie par John Yolton ou Philippe Hamou ${ }^{33}$. Reid discute précisément ces théories en raison de leur risque sceptique, mais il lutte aussi contre d'autres formes d'analogies trompeuses.

\section{Le modèle optique}

Toute opération intellectuelle n'est pas une perception consistant à percevoir une image mentale. Appelons le modèle critiqué ici, parce qu'il s'appuie sur des découvertes optiques, le modèle perceptif optique. Ce premier modèle $(M)$ peut se résumer en trois thèses qui s'imbriquent comme des poupées gigognes :

Thèse 1 : voir c'est percevoir l'image, rétinienne ou cérébrale, étudiée par la physiologie de l'œil.

Thèse 2 : toute perception consiste à percevoir une image sur ce modèle - lequel, de ce fait, devient le modèle optique de toute perception (y compris auditive, olfactive, etc.)

Thèse 3 : toute opération intellectuelle porte sur de simples images, thèse au centre de ce que l'on peut nommer le modèle optique perceptif de toute opération intellectuelle.

À la première thèse, Reid répond par la critique suivante.

33. J. W. Yolton, Perceptual Acquaintance from Descartes to Reid (en particulier p. 124125 , sur le lien entre le modèle optique et le risque sceptique) et Ph. Hamou, "Sensorium dei. Perception et présence spatiale de l'esprit dans la métaphysique newtonienne » in Revue philosophique de la France et de l'Étranger, 2014/1, n 139. 
Nous ne pouvons donner aucune raison qui explique pourquoi l'image sur la rétine doive être suivie de vision, alors qu'une image semblable sur toute autre partie du corps ne produit rien de tel que la vision (EIP, II.4, p. 95).

Sur ce point, la conviction de Reid est que l'image rétinienne est un fait que doit certes décrire la physiologie de l'œil (ainsi que l'a fait Porterfield), mais un fait de nature: nous ne pouvons pas rendre raison du lien quelle a avec la vision. Il faut admettre que pour voir, l'esprit humain a besoin d'une image rétinienne. De la même façon l'esprit humain a besoin d'une sensation de dureté pour percevoir une qualité objective de solidité. Ce sont là des faits dont la raison nous échappe. Et ainsi Reid peut élargir sa critique au point 2 . La référence à une image pour expliquer toute autre perception que visuelle paraît indue. Nous ne savons déjà pas, en effet, pourquoi une image rétinienne nous fait voir ; nous comprenons moins encore pourquoi nous aurions besoin d'une image pour avoir froid, sentir qu'un corps est dur, entendre un son ou goûter une saveur ${ }^{34}$.

Le modèle perceptif de la perception (thèse 2.) est donc trompeur. Déjà un peu avant ce passage, Reid avait nié que la métaphore de la "salle d'audience " (chamber of presence) ou encore celle de la camera obscura soient pertinentes pour la perception. La traduction de chamber of presence par " salle d'audience " a une acception juridique ou politique, celle d'une comparution qui précède le jugement, et elle s'accompagne en français d'une connotation auditive. Or la métaphore connote plutôt pour Reid un modèle optique de la perception (modèle, donc, où toute perception est perception d'une image).

Il y a une autre conclusion tirée des impressions qui sont faites sur le cerveau dans la perception, qui, je crois, n'a point de fondement solide, quoiqu'elle ait été adoptée très généralement par les philosophes. C'est celle selon laquelle des images de l'objet perçu sont formées par les impressions faites sur le cerveau, et l'esprit étant logé dans le cerveau comme dans une salle d'audience (chamber of presence), ne perçoit immédiatement que ces images, et n'a de perception de l'objet externe que par elles. Cette idée d'après laquelle nous percevons les objets externes non pas immédiatement mais en certaines images ou espèces fournies par les sens, semble être l'hypothèse philosophique la plus ancienne dont nous disposons sur le sujet de la perception, et avoir avec de légères variations conservé son autorité jusqu'à ce jour (IP, II.4, p. 90).

Reid se croit autorisé à rapprocher la métaphore lockienne de la salle d'audience et l'hypothèse newtonienne d'un sensorium, en raison du rôle que Locke donnait, dans ce passage, au cerveau. Mais il en donne une interprétation optique peut-être plus forte que Newton ne le faisait. L'hypothèse newtonienne du sensorium a certes été inspirée par les recherches optiques et la mise en évidence des images cérébrales dans la perception visuelle mais, au travers du concept de présence, elle renvoie aussi bien à une forme de

34. EIP, II.4, p. 95. 
majesté et d'empire. Pour Newton, la perception consiste en une coprésence de l'âme et de l'image cérébrale - analogue de la présence de Dieu au monde ou encore de la présence d'un roi en chaque lieu de son empire. En indiquant que cette coprésence est conçue par Newton comme une perception mentale, Reid procède à une sorte de rationalisation scientifique de ce qui restait encore assez métaphorique, voire métaphysique, chez Newton ${ }^{35}$. La radicalisation optique de l'hypothèse newtonienne s'accompagne ici d'une interprétation cérébrale des idées de la camera obscura de Locke, lesquelles, enfin, parce qu'elles sont des images (images), sont tenues pour des avatars des ombres de la caverne de Platon ${ }^{36}$.

La conséquence logique de ce modèle est que l'esprit perçoit l'image mais pas (ou pas directement) l'objet réel - ce qui est contraire à ce dont nous avons conscience ${ }^{37}$. Le glissement vers le modèle perceptif de nos opérations mine alors toute philosophie des idées - tout simplement parce que leurs objets sont suspectés d'être des apparences. Cela est, d'après Reid, porté à son comble par Hume lorsque ce dernier déclare que « la table que nous voyons semble diminuer à mesure que nous nous en éloignons; mais la table réelle, qui existe indépendamment de nous, ne souffre aucun changement " (Hume, $E E H$, xii, cité par Reid p. 178). Reid y oppose la critique suivante. Les apparences peuvent changer selon des règles géométriques sans que l'on doive nier que je vois la table réelle - plus exactement, les apparences changeant selon des rapports réglés et géométriques (par exemple la taille par rapport à la distance), nous pouvons juger des propriétés réelles et nous apprenons à le faire si promptement et habituellement que ce jugement devient une perception acquise. L'argument de Hume était proprement sceptique - hérité des tropes d'Énésidème. Il concluait que nous ne percevons jamais la table réelle parce que nous ne l'avons jamais à l'esprit. Le contre-argument de Reid consiste à dire que l'objet de la perception n'est pas interne précisément parce que les apparences changent selon des règles et des proportions géométriques - ce qu'elles ne feraient probablement pas si nous ne percevions directement que des images sans ancrage réel, ou encore si les apparences visibles ne signifiaient pas le réel.

[A] partir de l'hypothèse contraire, à savoir que les objets de la vue sont internes, aucune explication ne peut être fournie au sujet de quelque apparence que ce soit, et

35. Sur le sens de l'hypothèse du sensorium chez Newton, $c f$. Ph. Hamou, art. cit.

36. Ce rapprochement conduit Reid à concevoir les idées de Locke comme des images cérébrales. Et c'est une lecture de Locke que les historiens des idées, de Frederick Beasley ( $A$ Search of Truth in the Science of the Human Mind, 1822) à John Yolton (Perceptual Acquaintance from Descartes to Reid, Minneapolis, University of Minnesota, 1984, p. 100) ont à la fois critiquée et imputée à Reid. On notera toutefois que ce dernier admet qu'il n'est pas évident de savoir si Locke " pensait avec Descartes et Newton que les images (images) dans le cerveau sont perçues par l'esprit qui est présent, ou si elles sont imprimées dans Pesprit lui-même "; en d'autres termes il n'est pas sûr, d'après Reid, que Locke ait donné un rôle au cerveau dans la perception mentale des images cérébrales (EIP, II.4, p. 93).

37. EIP, II.4, p. 9. 
aucune cause physique attribuée au fait qu'un objet visible puisse, dans un cas, avoir telle figure et telle grandeur apparentes plutôt qu'une autre (EIP, II.14, p. 183).

Dans un autre passage, Reid reproche à Locke d'avoir employé le mot perception et le mot idée pour désigner de «simples apparences ou perceptions dans nos esprits ", qui ne sont ni vraies ni fausses (Locke, Essai, II.xxxii.1 cité dans EIP, IV.1). Cet abus de langage, selon Reid, vient de la doctrine des idées qui réduit toutes les opérations mentales à des perceptions d'idées :

Ils [Les philosophes : Gassendi, Descartes, Locke, Berkeley et Hume] ont probablement été conduits à cette impropriété de langage par la doctrine commune touchant les idées, qui enseigne que la conception, la perception des sens et le souvenir ne sont que différentes façons de percevoir des idées dans notre esprit (EIP, IV.1, p. 298).

Si cette théorie des idées était vraie, la perception et la conception seraient indiscernables. Or si Reid concède que parfois il est difficile de savoir si l'on conçoit simplement quelque chose ou si on le perçoit (notamment dans le rêve, la fièvre et la folie) et que les enfants peuvent également faire la confusion, il souligne qu'un degré convenable d'entendement suffit à ne pas la faire. Les exemples qu'il donne étant les expériences acataleptiques traditionnelles, il faut entendre que le risque n'est pas de mal comprendre la conception, mais de mal comprendre la perception en distinguant mal son objet (la réalité sensible, que l'on confondrait avec des conceptions fantaisistes) ${ }^{38}$.

Sous le régime de $(\mathrm{M})$, toutefois, il y a encore un monde d'objets, qui est certes un monde d'apparences, mais que nous pouvons concevoir et où nous pouvons dégager des régularités, faire des prévisions, et agir. Or il y a un autre modèle perceptif incriminé par Reid lorsqu'il critique la confusion entre différentes opérations mentales effectuées par les philosophes, et notamment la confusion entre perception et conception, un modèle peu relevé par les commentateurs mais plus radical parce qu'il va remettre en question la distinction entre l'opération mentale et son objet.

\section{Le modèle tactile}

Selon Reid, l'usage que Hume fait des mots perception et d'impression engendre deux confusions. D'abord, puisqu'il donne le nom d'impression à toute sensation ou passion, Hume est conduit à identifier les passions et les émotions avec des perceptions. En outre, ce compatriote sceptique parle des perceptions de la mémoire ou des perceptions de l'imagination, ce qui est aussi

38. En concédant que les expériences du rêve et de la folie sont des expériences de confusion entre imagination et perception, Reid accorde peut-être plus qu'il ne le devrait. Car s'agit-il vraiment d'une confusion entre simple appréhension et perception? Dugald Stewart notera que si nous ne faisons que songer à quelque chose sans y croire, il n'y a qu'une simple appréhension, mais si le rêve est trompeur, c'est que nous croyons qu' existe ce dont nous rêvons. 
confus et absurde que de parler des sons de la vue ou des odeurs du toucher. Selon Reid, la différence entre opérations mentales doit être maintenue, tout comme la distinction entre l'opération mentale et son objet. Un tel abus de langage est étroitement lié à un modèle perceptif qui est plus envahissant que le modèle optique. En effet, comme on va le voir, il ne se limite pas aux opérations intellectuelles, mais prétend également expliquer les passions ; il est également plus dirimant parce qu'il conduit à effacer la distinction entre acte et objet qui pouvait encore être maintenue au sein du modèle optique, de sorte que la seule existence susceptible d'être affirmée est réduite à une réalité mentale. Ce nouveau modèle - appelons-le $\left(M^{\prime}\right)$ - imbrique lui aussi trois thèses gigognes, dérivées cette fois d'un présupposé sur la sensation du toucher.

Thèse 1' : toucher c'est avoir une impression, un feeling où l'acte et l'objet se confondent.

Thèse 2' : toute perception consiste à avoir une telle impression ou un tel feeling.

Thèse 3' : toute opération de l'esprit se réduit à un feeling.

Le paradigme est manifestement tactile, puisque le sens fondamental est désormais le toucher. Toutefois, parce que la thèse 1'définit le toucher par un feeling ou une simple sensation, alors que la thèse 1 du modèle optique définissait la vue par la perception d'une image, ce modèle tactile devient sensitif et même sentimentaliste. Le toucher étant réduit à une pure sensation, en lui la distinction entre l'acte et l'objet disparaît. En outre, il est remarquable que la thèse 3', qui figure chez Hume d'après Reid, ne porte pas seulement sur les opérations intellectuelles (comme le faisait la thèse 3 du modèle optique) : dans ce modèle, la passion et les jugements de valeurs seront également, selon Reid, réduits à des feeling.

En fait, $\left(M^{\prime}\right)$ ne devient humien que dans sa thèse finale et totale. On découvre d'abord ce modèle, en II.14, sous la plume de Reid, comme une explication du présupposé arbitraire donnant lieu au modèle optique (impliquant un réalisme indirect). Lorigine du préjugé selon lequel la perception suppose une contiguïté (compris dans l'hypothèse d'un sensorium) est, nous dit alors Reid, l'analogie de toutes nos opérations mentales avec (M'). D'une certaine manière dans le modèle optique de Newton, il restait un présupposé métaphysique (le fait que la perception se fait par une coprésence), lequel s'explique par le modèle tactile (à savoir la perception par contact). Et c'est ainsi que Reid montre comment ( $M$ ') opère dans la "théorie des idées " pour étayer l'existence de ces intermédiaires idéels. La thèse centrale de Newton portait sur la perception visuelle. Or l'explication par l'hypothèse du sensorium où l'esprit serait présent à l'image perçue repose elle-même sur la thèse selon laquelle on perçoit par contact. Toute perception semble avoir besoin d'une impression mentale (thèse 2'). Reid ne nie d'ailleurs pas qu'il y a toujours une impression physique de l'objet sur le corps, sans laquelle nos sens externes ne fonctionnent pas. Mais en réalité il ne s'agit pas d'une 
impression mentale et cette impression ne saurait définir le feeling de la sensation ni même l'acte de percevoir l'objet externe ${ }^{39}$.

La généalogie des erreurs commises par ces modèles philosophiques s'explique par le constat récurrent qu'une impression corporelle se fait sur les organes des sens et la tendance à faire une analogie indue qui présuppose la contiguïté pour comprendre toute sensation et toute perception. La thèse 3 s'ensuit également (bien que dans la critique de la théorie des idées, les opérations mentales concernées semblent implicitement limitées aux opérations intellectuelles) :

Par le moyen des idées, toutes nos opérations mentales se résolvent en une seule opération : un espèce de sentiment (feeling) ou une perception immédiate des choses présentes qui sont en contact avec celui qui perçoit. Et sentir (feeling) est une opération si familière que nous pensons qu'elle ne requiert aucune explication et peut servir à expliquer les autres (EIP, II.14, p. 185).

Sous le régime de (M'), percevoir une idée c'est la sentir d'une certaine façon, et le sentir lui-même est l'opération mentale universelle. Toute opération se réduit à une perception qui n'est qu' un feeling. Hume est ici la cible, chez qui l'impression perd toute référence à une causalité extra-mentale et qui renoue avec le sens sceptique de la phantasia contre le sens mécanique du fantasme hobbesien. Bien que Reid puisse s'appuyer sur un ensemble de textes chez Hume allant en ce sens, notre propos n'est pas ici d'évaluer la pertinence ou la justesse de la lecture que celui-là fait de celui-ci ${ }^{40}$. Nous nous bornons à élucider sa critique, laquelle découle de trois réquisits. D'abord il faut éviter de réduire la diversité des opérations mentales : chacune est une expérience bien particulière et l'on ne peut pas généraliser au-delà de ces genres d'opérations que sont la perception, le souvenir, etc. : la description peut en venir à des faits originels mais chacun pour chaque genre. Hume qui a réduit la différence de genre de nos opérations mentales à une différence de degré (de vivacité) ou encore une différence de feeling, a échoué sur ce point. Il ne faut pas en faire des modes du sentir car le sentir n'est pas plus explicable que les autres.

39. L'impression, au sens commun (et non selon l'usage philosophique qui est un abus de langage) désigne le « changement produit dans un corps par l'opération de quelque cause externe ", ou par extension "le changement produit dans quelque sujet passif par l'opération d'une cause externe " (EIP, I.1, p. 35). Or l'esprit n'est pas passif dans l'opération mentale : "Si nous supposons qu'un être actif produit un quelconque changement en lui-même par son propre pouvoir actif, ceci ne saurait être nommé une impression. C'est l'acte ou l'opération de 'être lui-même, non une impression sur lui. De là il ressort que donner le nom d'impression à un effet produit en l'esprit c'est supposer que l'esprit n'agit pas du tout dans la production de cet effet. Si voir, entendre, désirer, vouloir sont des opérations de l'esprit, ils ne sauraient pas être des impressions. Si ce sont des impressions, ils ne sauraient être des opérations de l'esprit " (EIP, I.1, p. 35).

40. Sur le feeling, cf. Hume, Appendice au Traité de la nature humaine, \$9 SBN 627, tr. fr. in Hume. L'entendement, Paris, GF, 1995, p. 375-376; Enquête sur l'entendement humain, V.ii, tr. fr. in Essais et traités, Paris, Vrin, 2004, vol. 3, p. 85. Sur l'absence de considération concernant les causes de l'impression, cf. Traité de la nature humaine, I.i.2 et I.iii.5, p. 146. 
Mais ce sentiment (feeling) ou cette perception immédiate est aussi difficile à comprendre que les choses que nous prétendons expliquer par là. Deux choses peuvent être en contact sans aucun sentiment (feeling) ni perception. Il doit par conséquent exister chez celui qui perçoit un pouvoir de sentir ou de percevoir. Comment ce pouvoir est produit et comment il opère, voilà qui est bien au-delà de ce que nous pouvons savoir. Nous savons tout aussi peu si ce pouvoir doit être limité aux choses présentes et en contact avec nous. Aucun homme ne peut non plus prétendre prouver que l'Être qui nous a donné le pouvoir de percevoir les choses présentes ne puisse pas nous donner le pouvoir de percevoir les choses à distance, de nous souvenir des choses passées et de concevoir les choses qui n’ont jamais existé (ibid.).

Reid ne va pas montrer que le sentir est une opération qui pourrait être analysable en d'autres opérations plus primitives. Au contraire, il va montrer qu'en son genre le sentir est aussi "inanalysable » que les autres opérations - et que ce faisant il ne peut pas être un modèle pour expliquer les autres. Le contact en particulier n'explique pas plus le feeling que l'image rétinienne n'expliquait la vision. Ce sont des conditions de fait, universelles (et à ce titre des faits de nature) - mais dont la connexion nécessaire avec le phénomène du feeling ou de la vision nous échappe.

Il faut enfin souligner une différence entre le feeling et les autres opérations mentales : le feeling n'a pas d'objet distinct de l'acte même : ce qui est senti (car le langage parle bien en ce sens d'un objet de sensation) n'est pas distinct de la manière de le sentir. Le langage peut bien parler d'un objet de sensation (ce qui est senti) et de l'acte de sentir, il reste que ce qui est senti n'est pas différent de la sensation ou du sentiment (en somme, du sentir même). Les sensations peuvent certes être des objets pour d'autres sentiments (par exemple la sensation de plaisir ou de peine pour le désir et l'aversion), mais il ne faut pas confondre sensation et perception sous peine de négliger le fait que l'objet de la perception est externe, ce qui est typiquement l'erreur de Hume aux yeux de Reid ${ }^{41}$. Ainsi dans (M'), non seulement l'objet est immanent à l'acte, mais il se confond avec lui à tel point que toute ontologie extra-mentale est rendue impossible.

Dans une lettre adressée à Lord Kames, datée du $1^{\text {er }}$ décembre 1778, Reid avait déjà formulé ses deux principaux griefs contre le modèle perceptif de Hume : la réduction des opérations mentales à un seul genre, perceptif, et la confusion entre l'opération mentale et son objet ${ }^{42}$, corrélative d'une confusion particulière entre la perception et la conception. De manière intéressante, Reid avait commencé par concéder que l'on puisse parfois parler de nos opérations mentales comme de perceptions. Il existe, admettait-il, un sens figuré où l'on emploie le verbe percevoir, par exemple lorsque l'on dit que l'on perçoit la force d'une démonstration. Dans toute opération mentale par où se forme une croyance fondée sur une évidence aussi forte que la

41. EIP, I.1, p. 33.

42. The Correspondance of Thomas Reid, op. cit., p. 106-109. 
perception, on pourrait parler de perception au sens figurét ${ }^{43}$. Toutefois Reid ne craignait pas que ce sens figuré, très commun, n'induise un modèle perceptif de nos opérations mentales. Tant qu'il est figuré, cet usage " par extension " ne fait pas problème. En revanche, Reid épinglait déjà dans cette lettre la thèse humienne faisant de toute croyance une perception, thèse découlant selon lui d'une double confusion induite par l'usage du terme perception.

Elle [la croyance] est un ingrédient ou si vous préférez l'appeler ainsi un concomitant constant de la perception, de la mémoire, de la conscience, du jugement, du raisonnement. Mais je crois qu'il est très impropre de la nommer perception.

Je suis d'accord avec Monseigneur qu'on ne peut rien imaginer de plus absurde que la doctrine de David Hume sur ce sujet. Je suis prêt à penser qu'il y fut conduit, d'abord en donnant le nom de perception à toutes les opérations de l'esprit. De là, il s'ensuit nécessairement que la croyance est une perception, ou une modification de la perception. Deuxièmement, en confondant les opérations de l'esprit avec leurs objets. Lorsque je perçois, je dois percevoir quelque chose. Lorsque je crois, je dois croire quelque chose. Mais dans ce système, l'esprit qui perçoit ou qui croit, l'acte de percevoir ou de croire, \& l'objet perçu ou cru sont une même chose et appelés d'un seul nom (Reid, Lettre à Kames $1^{\text {er }}$ décembre 1778, op. cit., p. 109).

Toute croyance s'accompagne d'une conception de quelque chose. Pour autant, la croyance n'est pas une modification de la conception car la conception est seulement l'acte de penser à quelque chose alors que la croyance est une affirmation ou une négation, susceptible d'être vraie ou fausse. Déjà dans les Recherches, Reid avait fustigé la définition humienne de la croyance et « cette découverte " selon laquelle la sensation, la mémoire, la croyance et l'imagination, quand elles ont le même objet, ne sont que différents degrés de force et de vivacité dans l'idée :

Supposez que cette idée soit celle d'un état futur après la mort et qu'un homme y croie fermement : cela ne signifie rien de plus sinon quil en a une idée forte et vive. Un autre ne croit ni ne croit pas ; cela veut dire qu'il a une idée faible et éteinte. Supposez maintenant qu'un troisième homme croie fermement qu'il n'y a rien de tel : je suis bien en peine alors de savoir si son idée est éteinte ou vive; si elle est éteinte, alors il peut y avoir une croyance ferme quand l'idée est éteinte; si l'idée est vive, alors la croyance en un état futur et la croyance contraire ne doivent faire qu'une seule et même chose (REH, II.5, p. 30-31 $)^{44}$.

Les choses conçues ont beau être différentes, la théorie humienne se rend incapable de discerner les actes, et de distinguer ces actes de leur objet. Plus grave encore, la différence, pour un même objet, entre y croire, ne pas y

43. La conscience ou la mémoire, par exemple, ont une autorité aussi « certaine et irrésistible que celle des sens » (IP, II.5, p. 96).

44. Sur la critique par Reid de la définition humienne de la croyance, cf. également Thomas Reid, Recherches sur l'entendement humain d'après les principes du sens commun, II.5, trad. fr. par M. Malherbe, Paris, Vrin, 2012, p. 48-49. 
croire ou croire qu'il n'existe pas disparait. L'indiscernabilité entre imagination et perception est impliquée par la théorie des idées et en particulier par la réduction de toute opération à une perception sensible ou une conception sentie $(f e l t)$ de telle ou telle manière, laquelle vide la notion de croyance de son sens selon Reid. Le scepticisme est à ses yeux, donc, une conséquence des modèles philosophiques (M) et (M') plutôt que d'expériences soi-disant acataleptiques, telles que le rêve, dont on a vu qu'il est une simple conception et dont la confusion avec la perception n'est pas naturelle.

Ainsi donc, il ne faudrait pas réduire la cible de Reid au seul réalisme indirect impliqué par le modèle optique. Certes, ce dernier acquiert place et consistance par et dans l'histoire critique que Reid fait de la philosophie classique, de sorte que le rôle des idées chez Descartes et Locke sera lu au travers de ce prisme archéologique singulier. Toutefois en rejetant également (M') Reid dénonce un autre modèle sentimentaliste dont le subjectivisme est plus accompli encore ${ }^{45}$. Le souci épistémologique d'exclure un modèle où la vérité résulterait de la seule activité de l'esprit est ainsi indissociable des questions ontologiques et intentionnelles envisagées plus haut. L'esprit opère par les lois d'une efficace qui le transcende et l'évidence de ses opérations ne porte pas sur des objets ou des effets immanents. Cela anime sa critique de $\left(M^{\prime}\right)$ et cela impose au lecteur une certaine prudence dans l'interprétation des principes de notre constitution mentale que Reid nomme « sens commun ». Une véritable logique de l'esprit doit décrire les opérations mentales en tant qu'elles discernent la vérité, non en tant qu'elles la produisent. À partir de nos diverses expériences d'évidence, une telle description dégage les faits les plus généraux en la matière. Et pour finir, il faut souligner qu'une telle anatomie de l'esprit exclut la quête d'un modèle valable pour toutes nos opérations mentales. Car les opérations mentales se distinguent selon le type d'évidence qu'elles procurent. Cela suppose de ne pas en faire des modalités d'un modèle. Ainsi la perception constitue une raison de croire d'un certain ordre, le souvenir fournit une raison d'un autre ordre, la conscience, le témoignage, la démonstration ou l'induction d'autres raisons encore. Il y a certes une structure sémiologique que l'on retrouve dans différentes opérations mentales selon Reid : la croyance perceptive comme la croyance testimoniale, par exemple naissent du fait que certains signes (les sensations pour la première, les propos pour la seconde) nous font concevoir d'autres choses. Mais la conscience, par exemple, n’opère pas par signes. Reid maintient donc une hétérogénéité des espèces d'évidence, qui autorise de les croiser et les

45. "The truth of any proposition cannot depend upon the constitution; we except propositions in which the mind or some of its powers or operations is the subject of the proposition. But here we ought to distinguish between the truth of a proposition and my discernment of that truth. When truths are immediately assented to by all men as they are apprehended without instruction or reasoning, it may I think be said with property that the power by which we perceive the truth of the propositions is a part of our constitution " ( Of constitution", op. cit., f. 2.). 
confronter pour faire progresser les enquêtes, mais sans les réduire les unes aux autres.

Claire ETChegaray

Université Paris-Ouest-Nanterre-La-Défense Institut de Recherches Philosophiques 